\title{
A sleep pattern analysis and visualization system to support people with early dementia
}

\author{
Hoda Nikamalfard, Huiru Zheng ${ }^{*}$, Haiying Wang, \\ Maurice Mulvenna, Paul McCullagh \\ School of Computing and Mathematics \\ University of Ulster \\ Belfast, United Kingdom \\ Nikamalfard-H@email.ulster.ac.uk, \{h.zheng", hy.wang, \\ md.mulvenna,pj.mccullagh\}@ulster.ac.uk
}

\author{
Paul Jeffers \\ Fold Telecare \\ Fold Housing Association \\ Belfast, United Kingdom \\ Paul.Jeffers@foldgroup.co.uk
}

\begin{abstract}
Sleep disturbances are among the most distressing of all Alzheimer's disease related symptoms. In some cases, however, the sleep disturbance may be a marker for early Alzheimer's disease. In this paper we present a sleep pattern detection and visualization system developed to support the monitoring of sleep patterns and assessment of sleep disturbances for people diagnosed with dementia, at the early stages of the disease. The system provides visual tool kits for caregivers to view the sleep profiles and for investigators to analyse sleep patterns based on sensory data gathered at users' home via the telecare service.
\end{abstract} care

Keywords: sleep pattern, dementia, visualization, night time

\section{INTRODUCTION}

Sleep disturbances are among the most distressing of all Alzheimer's disease (AD) related symptoms. Sleep disorders and disruptive nocturnal behaviours present both a significant clinical problem and a cause of increased stress for caregivers [1]. People with $\mathrm{AD}$ or dementia, may have the sleep disturbances, similar to other older and within the expected norms. However, in some cases, the sleep disturbances may be a predictive marker for people with early $\mathrm{AD}$ [2]. Researchers have shown that sleep-related problems generally increase as $\mathrm{AD}$ progresses [3]. The degree of the irregular sleep-wake pattern can reflect the degree of cognitive impairment [4]. As the disease progresses, more severe sleep disturbances develop, which affects the continuity of nighttime sleep, alertness during the daytime, and overall wellbeing. The assessment of sleep disturbances can help toward an accurate diagnosis and a better understanding of the cognitive condition of the person with dementia.

A number of research projects have monitored and assessed sleep quality from a range of very different perspectives. Three approaches have been reported to record the sleep disturbances: (1) report or diary; (2) polysomnography; and (3) assistive technology [5]. Using state-of-art assistive technology can provide a non-invasive, pervasive and objective monitoring and assessment solution. However, most of these systems are focused on sleep pattern recognition instead of long term monitoring and analysis of sleep profiles for assessment of sleep quality [6]. To provide support for people with early dementia, the NOCTURNAL project (http://www.nocturnalproject.co.uk/) [7] [8] has developed a night monitoring and support system for people with early dementia living independently. Clients' sleep patterns are monitoring using bed sensors and Passive InfraRed (PIR) sensors.

A number of measurements have been proposed to assess the quality of sleep, including objective and subjective variables, such as [9][10]: time in bed, total sleep time, sleep latency, number of nocturnal awakenings, length of nocturnal awakenings, and (self) evaluation of sleep-quality, etc.

Some of these measurements are calculated from the devices used in the projects, and some are recorded by the clients or carers, which can be subjective and may be inaccurate. Moreover, there is no robust sleep pattern analysis system available to analyze sleep quality over a long term in order to assess and monitor cognitive function and health condition.

In this research, the main interest is develop a pattern analysis and visualization system (PAViS) to monitor shifts in sleep pattern and detect unusual patterns in order to observe changes in the health condition of people with dementia [5]. The measurements on sleep quality, quantity and rhythm are used for the assessment the sleep patterns. Daily, weekly and monthly information on these three types of sleep assessment are visualized to promote understanding by end users, carers and researchers.

The remainder of this paper is organized as the follows: Section 2 briefly reviews the NOCTURNAL system and the data collection techniques used. Section 3 describes the measurement variables used in this study and the sleep pattern analysis and visualization system. Results and visualization are presented in Section 4. The paper is concluded by summary and a description of further work in Section 5.

\section{NCTURNAL PROJECT AND DATA COLLECTION}

The NOCTURNAL project aims to develop Night Optimised Care Technology for UseRs Needing Assisted Lifestyles. This project addresses the needs of people (the clients) at the early 
stages of dementia at night, as well as those of their families and carers. In the NOCTURNAL system, a set of sensors is installed to monitor and manage the client's activities at night.

\section{A. System Setting}

An example of NOCTURNAL system setting is illustrated in Figure : bed occupancy sensor, PIR sensors (in the bed room hall way, kitchen, bath room), door contact sensors, and kitchen appliance sensors. For the sleep monitoring, the data we used are collected from the bed occupancy sensors, bed room PIR, and hall way (outside of the bedroom) PIR, all 24 hours a day.

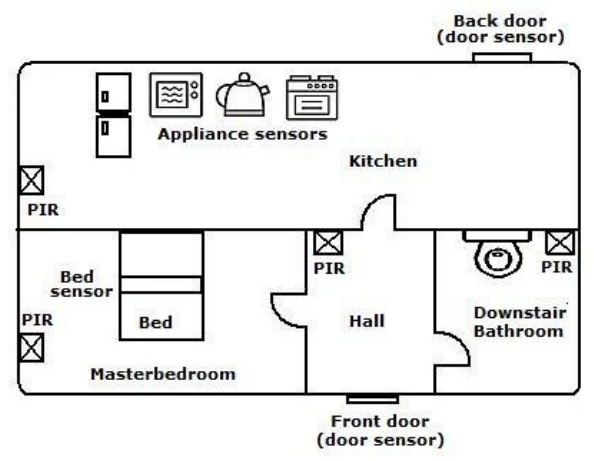

Figure 1 An example of NOCTURNAL system setup [5]

The participants in this study are elderly people with early stage dementia diagnosis who live independently, with telecare support, provided by the FOLD telecare. All the data used in this paper are collected from a single female client, Mrs. F, as an exploratory piece of research.

The data preprocessing in this study used the same rules as in [5]; readers are referred to this paper for a more detailed description.

\section{B. Sleep measurement varibles}

Three measures are used to monitor the shifts of sleep patterns and to assess the sleep changes [5], This is a set of parameters which try to quantify the different aspects of the resting periods. Taken together they provide a comprehensive indication of the effectiveness of the rest the client is experiencing.

1) Quantity of sleep

This variable tracks the total amount of time the person spends in bed during each 24 hour period starting at 12 midday each day. It may show a high degree of variation from day to day but the average trending over extended periods is a good indicator of how much rest the client is getting overall.

2) Quality of sleep

Quality of sleep is assessed by looking at the number (sleep episodes) and duration of periods spent in bed over the 24 hour period. Generally the fewer and more extended these are - the better the quality of the rest period.

3) Rhythm of sleep

This variable seeks to track this by visualising when the client take their rest period in the 24 hour period. This can change slowly so monitoring over weeks or months is required. Monitoring and attempting to support and stabilise this aspect is an important objective of the Nocturnal project.

\section{SYSTEM IMPLEMENTATION}

The sleep pattern detection and visualization system, PAViS, is implemented using Java. A MySQL database is used to store sensory data obtained from the NOCTURNAL system. The database contains time stamped data and each sensor's status.

\section{A. Architectural Framework}

In order to embrace flexibility and robustness, the system was designed using a three-tier architecture (Figure 2): presentation tier, application tier and data tier. The Presentation tier offers a user friendly interface while the Application tier controls functionalities and manipulates underlying logical connection of information flows; finally, the Data tier handles information storage and retrievals from database.

Presentation Tier: Visualization is one of the key issues in telecare systems as telecare staff and the clients' carers may not be fluent ICT users.

In this research, we provide several informative types of visual feedback on client's daily, weekly and monthly sleep information and profiles, including:

(1) Daily sleep pattern which displays a client's daily sleep - wake cycles;

(2) Weekly sleep pattern which includes:

- Client's daily sleep pattern over past 7 days. This section enables convenient visual comparison of sleep patterns and detection of unusual days;

- Summary and trend of seven days of daily sleep-wake episodes; and

- Summary and trend of seven days daily amount of sleep time.

(3) Monthly sleep pattern which includes:

- Summary and trend of four weeks of daily sleep-wake episodes; and

- Summary and trend of four weeks daily amount of sleep time.

These visual feedbacks reflects the quantity, quality and rhythm of the daily sleep pattern, and data can be selected over a chosen period of time.

Presentation tier is implemented using the Java libraries JFreeChart (http://jfree.org/jfreechart/) to visualize the information graphically.

Application Tier: This tier is the middle layer which bridges the gap between user interface and underlying database, hiding technical details from users of the system. Java classes in this tier receive requests coming from the presentation tier. By processing these requests, application tier classes decide which sensory data is needed from database in 
order to prepare requested graphs. After receiving sensory data from data tier, the application tier:

(1) Detects valid start and end points of each sleep episode;

(2) Calculates length of valid sleep episodes;

(3) Calculates number of valid sleep episodes;

(4) Calculates sleep trend line;

(5) Summarizes the sleep pattern of client;

(6) Groups data for 7 days or 4 weeks in an appropriate data structure to be used by the presentation tier.

Data Tier: Java classes in the data tier are responsible for

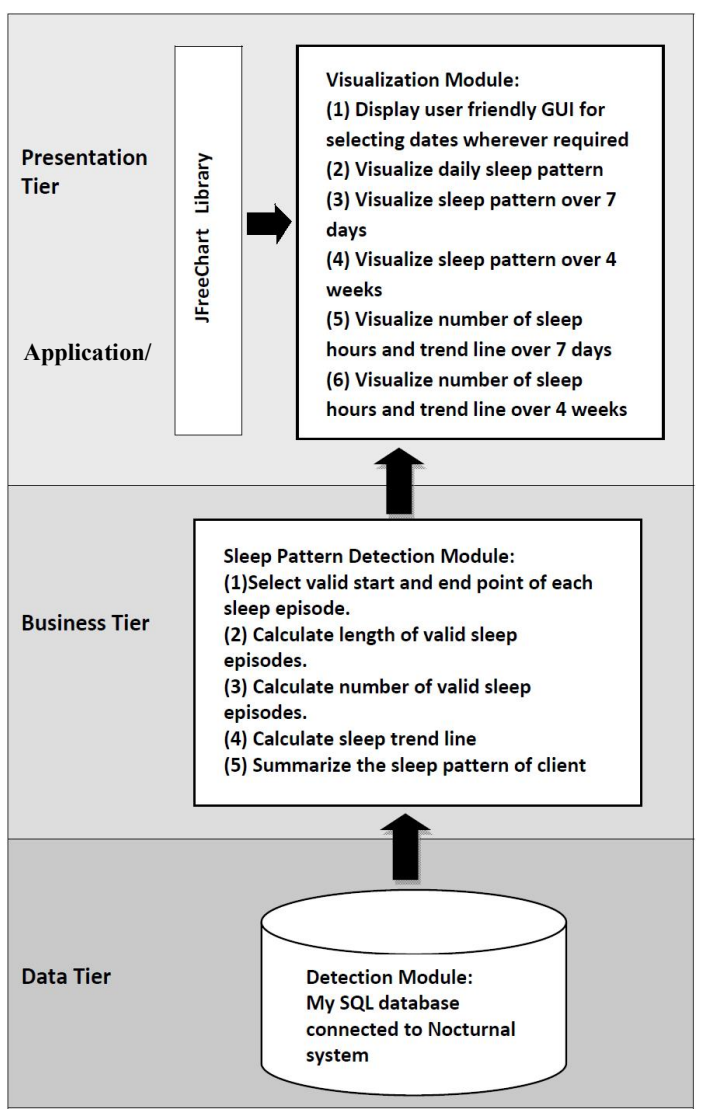

Figure 2. The architecture of the proposed Sleep Pattern Detection and Visualization system

handling requests of the application tier for sensory data. These classes use JDBC to access the MySQL database, which is connected to the NOCTURNAL system and contains sensory data.

\section{B. Graphical User Interface}

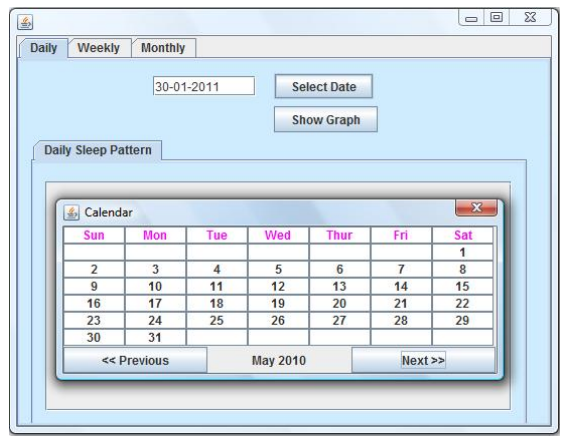

Figure 3. User friendly calendar opens by pressing "Select Date" button; after choosing desired date, the calendar closes and the selected date appears in the text box
PAViS provides a user friendly calendar for the user to select the desired date. The calendar shows valid days for which data is available in the NOCTURNAL database (Figure 3). The calendar feature is available for viewing a daily, weekly and monthly sleep profiles.

PAViS provides daily, weekly and monthly sets of graphical feedback or summary using an associated tab. To keep a consistent design theme different background colours are used, blue for daily information, green for weekly information and pink for monthly information.

Figure 4 visualizes the daily sleep pattern of the client on a date selected. The numbers of sleep episodes and total sleep time of the day are automatically calculated and displayed on the graph. The total sleep period (rhythm), the longest sleep period and the NOCTURNAL awaken (out of the bed) times are also clearly displayed. The system also provides a 'zoom in/ out' function to allow detailed analysis of the

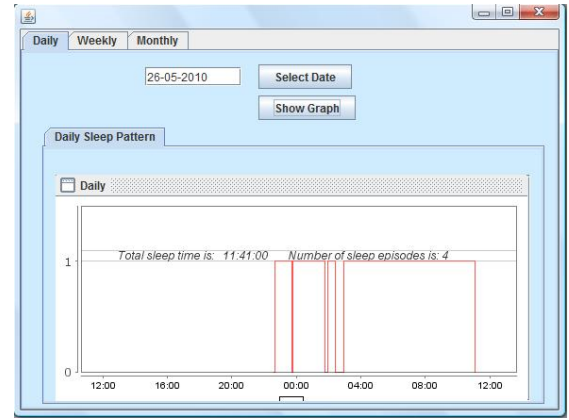

Figure 4. Summary of client's daily sleep pattern. The x-axis represents the time, from 12.00 o'clock to the next 12.00 o'clock. The $y$-axis indicates the sleep status: ' 0 ' is out of bed, and ' 1 ' is in bed. Selected date is shown in text box. Total sleep hours and number of sleep episodes are shown above the graph.

events.

As illustrated in Figures 5, the weekly tab contains three subtabs for three types of weekly sleep information: sleep pattern; sleep hours and sleep episodes. In order to view these three types of information on a desired week, the user selects first day of that week from the calendar. By pressing "Show Graph" button, the associated graphs of selected week will appear on

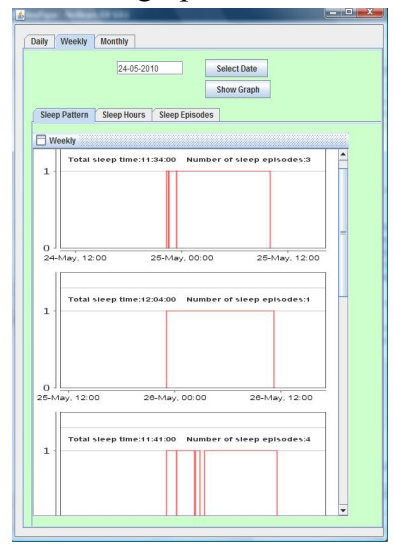

Figure 5. An illustration of a client's weekly sleep pattern. The $\mathrm{x}$-axis is the time in 24 hours from 12.00 o'clock to 
their internal tabs. The first tab displays sleep pattern for seven days starting from selected date; the second tab shows summary of total sleep amounts over seven days and third tab displays summary of daily sleep episodes over seven days.

Similar to the weekly sleep profile, the monthly sleep information provides four consecutive weeks of sleep pattern,

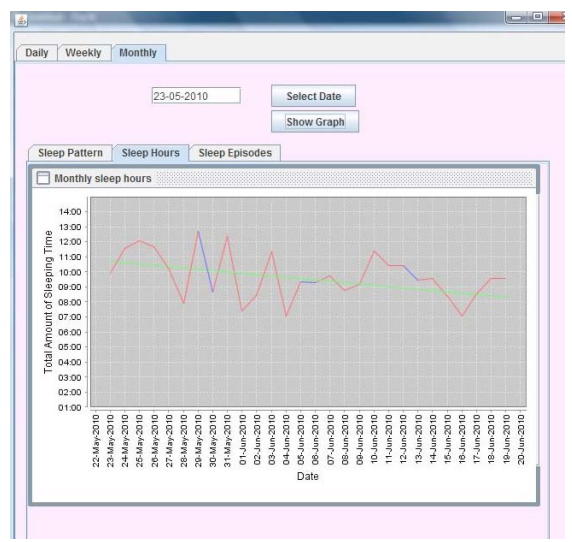

Figure 6 An illustration of a client's monthly sleep hours. The xaxis is the date. The y-axis indicates the total sleep time in hours.

The straight line in the middle indicates the trend of the sleep hour changes during the four weeks.

sleep hours and sleep episodes. Figure 6 visualizes the changes and trends of sleep hours over four weeks.

\section{SUMMARY AND DISCUSSION}

The PAViS is developed to provide a user-friendly platform for telecare users to analyze the patterns, changes and trend of clients' sleep quality and quantity. Three main functions are implemented to view: sleep patterns, sleep hours and sleep episodes on daily, weekly and monthly basis. Healthcare professionals can review clients' profile and compare the changes of trend on various period of time, which may be helpful in determine the cognitive impairment stages. Figure 5 shows an example of one day sleep pattern, although the client had good amount of sleep hour, there were 4 episodes observed, which indicate that the client had some sleep disturbances during the night. To find out if it was her normal pattern or abnormal pattern, the carer can use weekly view to compare that date with previous dates (Figure 5), and the trend of changes. Figure 6 shows a slightly decreasing of total sleep amount of the client in one week, while a better sleep quality (the trend of sleep episode was decreasing) was observed in another week.

While current telecare service focuses on providing telemonitoring of clients' daily activity, and tries to detect abnormal behavior, it is also important to investigate the correlation of behavior profile, such as sleep pattern profile, with the clients' health condition. The proposed PAViS system provides a tool to enable telecare service and carers to be able to have a better overview of the client's behaviour so as to provide sufficient support when necessary. For example, Figure 6 shows that the client had a decreasing daily sleep hour over the month; however, those days that the client had longer sleep hours and didn't indicate that she had better sleep pattern, because it may be caused by her staying longer in bed but also she may have more times of getting up.

Furthermore, the knowledge discovered or obtained from the long-term sleep profiles can also be used to support intervention in detecting and responding to abnormal sleep pattern. This can also provide objective feedback to people with earlier dementia and their carer for a better management of their condition. Further work will be carried out to investigate the above area while more data from different clients will be analyzed.

\section{Acknowledgment}

This research is supported by a research grant from the Technology Strategy Board and Engineering and Physical Sciences Research Council, UK (TS/G002452/1).

\section{References}

[1] D. Neef and D Larson, "Sleep and quality of life in Alzheimer's disease and the dementias", in Sleep and quality of life in clinical medicine, J. C. Verster, S. R. Pandi-Perumal and D. L. Streiner, Eds. Humana Press, 2008, pp. 155-160.

[2] C. Singer and A. Bahr, "Assessing and Treating Sleep Disturbances in Patients With Alzheimer's Disease", Psychiatric Times,vol. XXII (13), | November 2005.

[3] K.E. Moe, M.V. Vitiello, L.H. Larsen and P.N. Prinz, "Symposium: cognitive processes and sleep disturbances: sleep/wake patterns in Alzheimer's disease: relationships with cognition and function", J Sleep Res, 4(1), pp. 15-20.

[4] B. EI-Ad, A. Culebras, "Irregular sleep-wake pattern", Medlink Neurology, http://www.medlink.com/ medlinkcontent.asp, access on 01 Oct. 2010.

[5] H. Zheng et al, "Monitoring and Analysis of Sleep Pattern for People with Early Dementia", In 1st Workshop on Knowledge Engineering, Discovery and Dissemination in Health (KEDDH'10), in Proceedings of 2010 IEEE International Conference on Bioinformatics and Biomedicine Workshops, 18-21 December 2010 Hong Kong, pp. 405 - 410, China.

[6] A. A. P. Wai et al, "Situation-aware patient monitoring in and around the bed using multimodal sensing intellignece", in Proc. of 2010 Sixth International Conference on Intelligent Enviroments, pp. 128-133.

[7] H. Zheng et al, "NOCTURNAL: Night Optimised Care Technology for UseRs Needing Assisted Lifestyles". In: eChallenges 2009, Istanbul, Turky.

[8] P.J. McCullagh et al, "Nocturnal Sensing and Intervention for Assisted Living of People with Dementia", In Healthcare Sensor Networks Challenges Towards Practical Application, D. Lai, R. Begg and M. Palaniswami (Eds.), Taylor and Francis/CRC Press. 2010.

[9] A. G. Mayers, G. Andrew, J. C. VanHooff, and David S Baldwin, "Quantifying subjective assessment of sleep and life-quality in antidepressant-treated depressed patients". Human Psychopharmacology, vol. 18(1), pp. 21-27.

[10] G.E. Brannon, V. Subir and A. Gentill, "Sleep disorder,Geriatric", http://emedicine.medscape.com/article/292498-overview, access on $15^{\text {th }}$ Feb, 2011. 